\title{
A Collection of Results for the SPC/E Water Model
}

\section{R.D. Mountain}

\author{
U.S. DEPARTMENT OF COMMERCE \\ Technology Administration \\ National Institute of Standards \\ and Technology \\ Thermophysics Division \\ Chemical Science and Technology Laboratory \\ Gaithersburg, MD 20899
}

\section{A. Wallqvist}

Science Applications International Corporation Frederick Cancer Research and Development Center National Cancer Institute

Frederick, MD 21702

QC 



\title{
A Collection of Results for the SPC/E Water Model
}

\section{R.D. Mountain}

\author{
U.S. DEPARTMENT OF COMMERCE \\ Technology Administration \\ National Institute of Standards \\ and Technology \\ Thermophysics Division \\ Chemical Science and Technology Laboratory \\ Gaithersburg, MD 20899
}

\section{A. Wallqvist}

Science Applications International Corporation Frederick Cancer Research and Development Center National Cancer Institute

Frederick, MD 21702

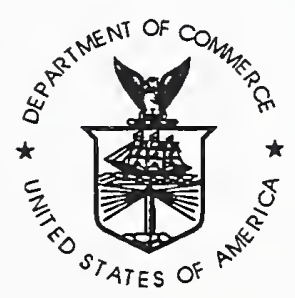

U.S. DEPARTMENT OF COMMERCE Ronald $H$. Brown, Secretary

TECHNOLOGY ADMINISTRATION Mary L. Good, Under Secretary for Technology

NATIONAL INSTITUTE OF STANDARDS AND TECHNOLOGY

Arati Prabhakar, Director 



\title{
A collection of results for the SPC/E water model
}

\author{
Raymond D. Mountain \\ Thermophysics Division \\ Chemical Science and Technology Laboratory \\ National Institute of Standards and Technology \\ Gaithersburg, MD 20899 \\ Anders Wallqvist \\ Science Applications International Corporation \\ Frederick Cancer Research and Development Center \\ National Cancer Institute \\ Frederick, MD 21702
}

\begin{abstract}
This report contains a compilation of molecular dynamics computer simulation results for the simple point charge extended model (SPC/E) for water. In addition to published results, this compilation contains unpublished results developed by the authors. The properties included are the static dielectric constant, the self-diffusion coefficient, the internal energy, and the pressure. The simulation values of the dielectric constant are compared with a new correlation based on available experimental values.
\end{abstract}

\section{Introduction}

The simple point charge extended (SPC/E) model $^{1}$ for water has proven to be one of the more robust, but simple of the many interaction models proposed for water since the early work of Stillinger and Rahman. ${ }^{2}$ In this note we present a survey of SPC/E results for the potential energy $U$, the self-diffusion coefficient $D_{S}$, and the dielectric constant $\epsilon$ over a wide range of temperatures and densities. No claim for completeness of this survey is made, nor for the validity of any specific result. There is reasonably good consistency between the results in various studies. This report is the outgrowth of some simulations performed by the authors. Also, experimental values of the dielectric constant are listed so that the extent to which this model mimics water can be examined.

The SPC/E model assumes that a water molecule is a rigid object with an intramolecular distance of $0.1 \mathrm{~nm}$ between oxygen and hydrogen interaction sites (O-H distance) and with an angle of $109.47^{\circ}$ between the $\mathrm{O}-\mathrm{H}$ bonds. The intermolecular site-site interactions are defined in terms of the distances between the sites. Although these sites are commonly interpreted in terms of oxygen and hydrogen atoms, they are properly only sites for atomatom and Coulomb interactions. There are partial charges assigned to the sites so as to mimic an effective charge distribution of a water molecule in liquid water. The charge on the oxygen site is $-0.8476 \mathrm{e}$ and the charge on the hydrogen site is $0.4238 \mathrm{e}$ with the result that the permanent dipole moment $\mu=7.84 \times 10^{-30} \mathrm{C} \mathrm{m}(2.35 \mathrm{D})$. The resulting charge distribution has a quadrupole moment (in units of $10^{-40} \mathrm{C} \mathrm{m}^{2}$ ) $\mathrm{Q}_{x x}$ of $7.290, \mathrm{Q}_{y y}$ of -6.286 and $Q_{z z}$ of -1.007 compared with the experimental values of $8.77,-8.34$, and -0.43 respectively. ${ }^{3}$ 
The permanent dipole moment is larger than the moment of an isolated molecule, $\mu_{0}=$ $6.17 \times 10^{-30} \mathrm{C} \mathrm{m}(1.85 \mathrm{D})$, and the induction energy associated with the excess dipole moment,

$$
E_{\text {ind }}=\frac{1}{2 \alpha} \sum_{j}\left(\mu_{j}-\mu_{0}\right)^{2}
$$

where $\alpha$ is the polarizability of the molecule, amounts to $5.213 \mathrm{~kJ} / \mathrm{mol}$. Inclusion of this mean-field value of the induction energy when the site charges were determined is the distinction between the original SPC model and the SPC/E model.

In addition to the Couloumb interaction between molecules, the model has a Lennard-Jones interaction,

$$
\phi(r)=4 \epsilon_{L J}\left[(\sigma / r)^{12}-(\sigma / r)^{6}\right]
$$

between the oxygen sites. The Lennard-Jones parameters are $\epsilon_{L J}=78.24 k_{B}$ and $\sigma=$ $0.31656 \mathrm{~nm}$. Here $k_{B}$ is Boltzmann's constant. The energy of the system is the sum of the mean-field induction energy, the Coulomb energy, and the Lennard-Jones energy.

First, the unpublished simulation results of the authors will be presented. Then, results of other studies will be listed. A summary discussion concludes this report.

\section{Molecular dynamics studies of SPC/E water}

A series of molecular dynamics simulations have been performed by RDM using the SPC/E model to determine the dielectric constant of this model at elevated temperatures and at densities below $1000 \mathrm{~kg} / \mathrm{m}^{3}$. The development of an extensive correlation ${ }^{4}$ of experimentally determined dielectric constants in this range of thermodynamic states was the motivating factor for this study. This correlation is based on data in the interval $230 K<T<873 K$ with densities on the order of $1000 \mathrm{~kg} / \mathrm{m}^{3}$ or less. This correlation reproduces the experimental values within \pm 0.5 with much narrower bounds for temperatures below the critical temperature. Our comparisons between simulation values and values provided by this correlation are limited to this range of temperatures and densities. If no correlation value is quoted, it is for this reason. If a value for the dielectric constant is obtained by an extrapolation outside the range of the data, that value is enclosed in parentheses, $(\ldots)$.

The simulations were performed on systems of 216 rigid water molecules contained in a cubic, periodically continued cell. Ewald summations ${ }^{5}$ with conducting conditions at infinity were utilized to account for the long range Coulomb interactions while the LennardJones interactions were truncated at one-half the box size. The equations of motion were integrated using an iterated Beeman algorithm ${ }^{6}$ and a time step of $10^{-15} \mathrm{~s}$. A quaternion representation ${ }^{7}$ was used for the orientational degrees of freedom. The temperature of the system was controlled by separate Nóse-Hoover thermostats ${ }^{8}$ for the translational and rotational degrees of freedom. The coupling constant between the thermostats and these degrees of freedom had a value $Q=0.05 m \sigma^{2}$ where $m$ is the mass of a water molecule and $\sigma$ is the Lennard-Jones parameter. This value provided a reasonably steady value for both the translational and rotational kinetic energies as the simulation progressed.

The properties reported here are the energy, self-diffusion coefficient, and the dielectric constant. A total of sixteen states have been simulated. The temperature and density pairs along with these properties are listed in Table 1. 
The duration of a given simulation was dictated by the long sampling times needed to obtain stable estimates for the dielectric constant, $\epsilon$, from the fluctuation expression ${ }^{5}$,

$$
\epsilon=1+\frac{4 \pi}{3} \frac{<\mathrm{M}^{2}>}{k_{B} T V} .
$$

Here, $<\mathbf{M}^{2}>$ is the ensemble average of the square of the total dipole moment of the system of molecules, $V$ is the volume, and $T$ is the absolute temperature. Since each configuration yields only one sample for $\mathbf{M}$ and the decay of the correlations of the total moment is a relatively slow process, requiring tens of picoseconds, with the result that the simulation times needed range from 100 ps-1000 ps. ${ }^{9}$ The time step used in the integration of the equations of motion is $10^{-15} \mathrm{~s}$. Thus several hundred thousand iterations of the equations of motion are necessary for each thermodynamic state.

The values of the dielectric constants listed in Table 1 have converged to within \pm 1 unit. This conclusion is based on the fluctuations in the long averaging time estimates of $<\mathbf{M}^{2}>$ with increasing averaging time. The absence of any apparent drift in the average was taken as a necessary condition for convergence. The self-diffusion coefficients were estimated using the Einstein relation for the long-time behavior of the mean-square displacement of molecules,

$$
<[\Delta r]_{t}^{2}>\rightarrow 6 D_{S} t
$$

where

$$
<[\Delta r]_{t}^{2}>=<\frac{1}{N} \sum_{j=1}^{N}\left[\mathbf{r}_{j}(t)-\mathbf{r}_{j}(0)\right]^{2}>
$$

and the angle brackets $\langle\ldots\rangle$ indicate an average over the enclosed quantity.

A second set of simulations was performed in the supercritical region by AW. These simulations employed 216 rigid water molecules enclosed in a cubic, periodic cell under constant volume conditions. The long-range electrostatic interactions were include via an Ewald simulation, whereas the Lennard-Jones interactions were spherically truncated at $0.93 \mathrm{~nm}$. The equation of motions were integrated using a velocity Verlet algorithm coupled to a SHAKE/RATTLE procedure ${ }^{5}$ to maintain the intramolecular water geometry. A timestep of $2.0 \mathrm{fs}$ was used, velocities were periodically rescaled to maintain a constant temperature and the translational and rotational temperatures were monitored to avoid any imbalances between these degrees of freedom. After a 20 ps equilibration period the systems were sampled for an additional 400 ps during which the ensemble averages were calculated. The results from these simulations are listed in Table 2. No uncertainty estimates have been made for these results.

\section{Results from other simulation studies of SPC/E water}

The properties of the SPC/E model have been examined for a wide range of densities and temperatures. A systematic examination of the density and temperature variation of the dielectric constant of water has been reported recently. ${ }^{10}$ The results of this study are listed in Table 3. The results of other studies for the dielectric constant, for the potential energy (including the induction energy $E_{\text {ind }}$ ), and for the self-diffusion coefficient are listed in Tables 4 and 5, along with the references for the source of the values. Table 4 contains 
dielectric constant results, Table 5 contains potential energy results. Diffusion coefficient results are found in Tables 4 and 5 .

The results from Ref. 13 lie along the liquid vapor coexistence curve for the SPC/E model. The temperature-density trajectory determined there does not exactly coincide with that of water, with the largest deviation of the model density on the liquid side being about $15 \%$ below that of the liquid near the critical point. The deviations on the vapor side are large with the model density greater than the density of water vapor.

The long-range Coulomb interaction has been treated in various ways. In the simulations leading to Tables 1 and 2, Ewald summation methods were used. This is generally agreed to be a "correct" method, although it is computationally expensive. Alternative formulations, such as reaction-field methods can yield good results if used judiciously. Truncation, with or without smoothing, tends not to produce the proper structure in the liquid. This is particularly troublesome for dielectric constant determinations.

In addition to results summarized in Tables 3,4 , and 5 , the surface tension of SPC/E water has been determined with reasonably good agreement with experimental values between $316 \mathrm{~K}$ and $573 \mathrm{~K} .{ }^{19}$. There is also one report of the shear viscosity at $277 \mathrm{~K}$ and at $300 \mathrm{~K} .{ }^{16}$

\section{Discussion}

Since the simulation results listed in Table 1 were motivated by interest in the high temperature values for the dielectric constant, it is appropriate to examine that body of results in more detail. The density-temperature values for which SPC/E values of $\epsilon$ have been reported are indicated in Fig. 1. The temperature variation of the dielectric constant is indicated in Fig. 2 where a dashed line joins values on the coexistence curve reported in Ref. 13 and solid lines join points of constant density. A logarithmic scale is used to avoid visual clutter. There is fairly good consistency between the values obtained by various workers for states with temperatures greater than $400 \mathrm{~K}$. There are however, some considerable discrepancies reported for lower temperatures (and necessarily higher densities). The discrepancies occur in the region where one must be careful to obtain adequate sampling for the total moment fluctuations, $\left\langle\mathrm{M}^{2}\right\rangle$. In general, the SPC/E values for the dielectric constant are lower than the experimental values obtained at the same density and temperature. This is indicated in Fig. 3 where the relative deviation of the simulated value of the dielectric constant from the value provided by the data correlation of Ref. 4 . Figs. 4 and 5 display the relative deviations along isochores and isotherms, respectively.

The self-diffusion coefficients and potential energy values for the SPC/E model are collected in "one place". No attempt has been made to compare the calculated and experimental values for these quantities.

All of the results reported here are based on classical dynamics simulations. Since the de Broglie wavelength of a hydrogen atom at $300 \mathrm{~K}$ is on the order of $0.1 \mathrm{~nm}$, quantum dynamical effects can be expected to be important. This has been shown to be the case for room temperature. ${ }^{20}$ It is noteworthy that the isotopic mass effect on the self-diffusion coefficient for hydrogen, deuterium, and tritium ${ }^{12}$ is smaller than the quantum dynamical effect.

\section{Acknowledgment}

We wish to thank the authors of Ref. 4 for making their correlation available to us prior to publication. Any extrapolations indicated in the tables are not their responsibility. 


\section{References}

1. H. J. C. Berendsen, J. R. Grigera, and T. P. Straatsma, J. Phys. Chem. 91, 6269 (1987).

2. F. H. Stillinger and A. Rahman, J. Chem. Phys. 60, 1545 (1974).

3. C. G. Gray and K. E. Gubbins, Theory of Molecular Fluids. Volume 1: Fundamentals, (Clarendon Press, Oxford, 1984), p. 582. Units are discussed on p. 575. Dipole moment: $1 D=3.335 \times 10^{-30} \mathrm{C} \mathrm{m}$. Quadrupole moment: $1 \times 10^{-26} \mathrm{esu}=3.335 \times 10^{-40} \mathrm{C} \mathrm{m}^{2}$.

4. D. P. Fernández, A. R. H. Goodwin, E. W. Lemmon, J. M. H. Levelt Sengers, and R. C. Williams, J. Phys. Chem. Ref. Data (1996), in preparation.

5. M. P. Allen and D. J. Tildesley, Computer Simulation of Liquids, (Clarendon Press, Oxford, 1989).

6. D. Beeman, J. Comp. Phys. 20, 130 (1976).

7. D. J. Evans and S. Murad, Mol. Phys. 34, 327 1977). Quaternion based equations of motion for nonspherical top molecules are discussed by R. Sonnenschein, J. Comp. Phys. 59, 347 (1985) and by D. C. Rapaport, ibid 60, 306 (1988). It is essential to use the Euler equations for nonspherical objects. Failure to do this leads to incorrect values for the self-diffusion coefficient. D. M. Heyes, J. Chem. Soc. Faraday Trans. 90, 3039 (1994).

8. G. J. Martyna, M. L. Klein, and M. Tuckerman, J. Chem. Phys. 97, 2635 (1992). This article contains a concise description of the use of thermostats in molecular dynamics simulations.

9. R. D. Mountain and D. Thirumalai, Computer Phys. Comm, 62, 352 (1991).

10. M .Neumann, in Physical Chemistry of Aqueous Systems, H. J. White, Jr., J. V. Sengers, D. B. Neumann, and J. C. Bellows, eds. (Begell House, New York, 1995), pp.261-268.

11. I. M. Svishchev and P. G. Kusalik, J. Chem. Phys. 99, 3049 (1993).

12. I. M. Svishchev and P. G. Kusalik, J. Phys. Chem. 98, 728 (1994).

13. Y. Guissani and B. Guillot, J. Chem. Phys. 98, 8221 (1993), and B. Guillot and Y. Guissani, J. Chem. Phys. 99, 8075 (1993).

14. E. Wasserman, B. Wood, and J. Brodholt, Ber. Bunsenges. Phys. Chem. 98, 906 (1994).

15. L. A. Báez and P. Clancy, J. Chem. Phys. 101, 8937 (1994).

16. P. E. Smith and W. F. van Gunsteren, Chem. Phys. Letters 215, 315 (1993).

17. I. I. Vaisman, L. Perera, and M. Berkowitz, J. Chem. Phys. 98, 9859 (1993).

18. J. Brodholt and B. Wood, J. Geophys. Res. 98, 519 (1993).

19. J. Alejandre, D. J. Tildesley, and G. A. Chapela, J. Chem. Phys. 102, 4574 (1995).

20. S. R. Billeter, P. M. King, and W. F. van Gunsteren, J. Chem. Phys. 100, 6692 (1994). 


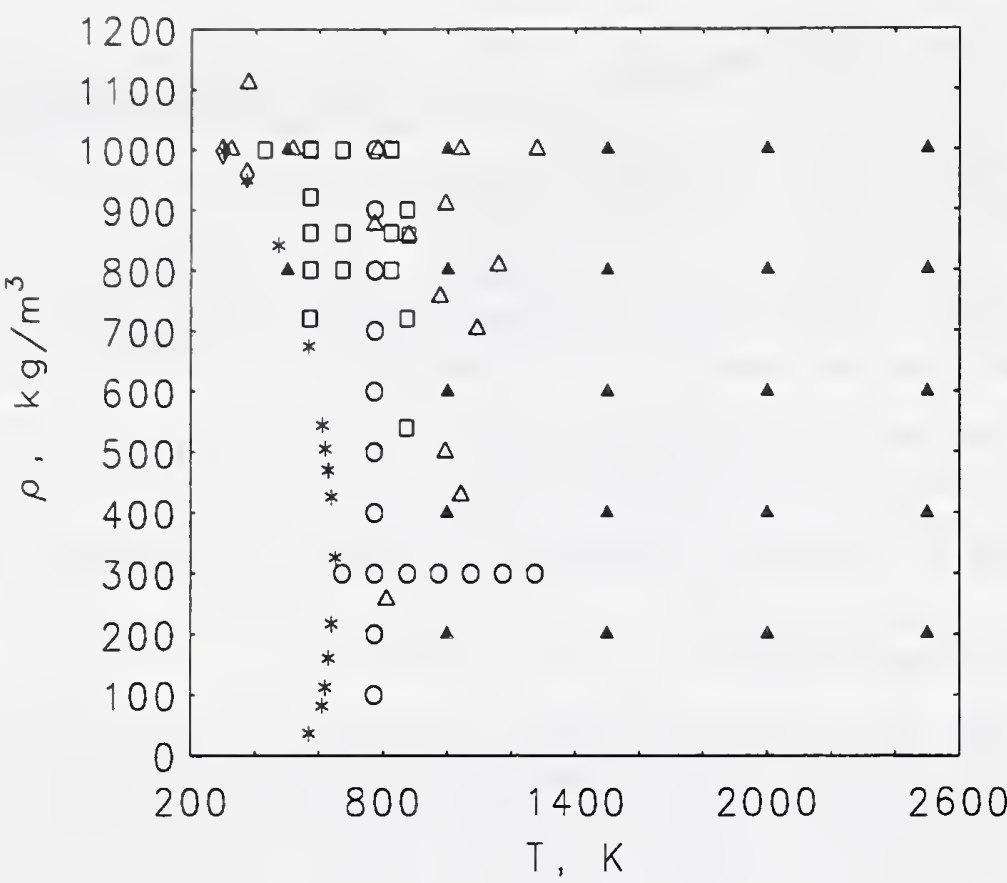

Fig.1 The density and temperature values for which the dielectric constant of the SPC/E model has been reported. The symbols indicate the source: square, Table 1 ; circle, Table 2 ; *, Ref. 13; open triangle, Ref. 14; solid triangle, Ref 10; and diamond, Refs. 11 and 12.

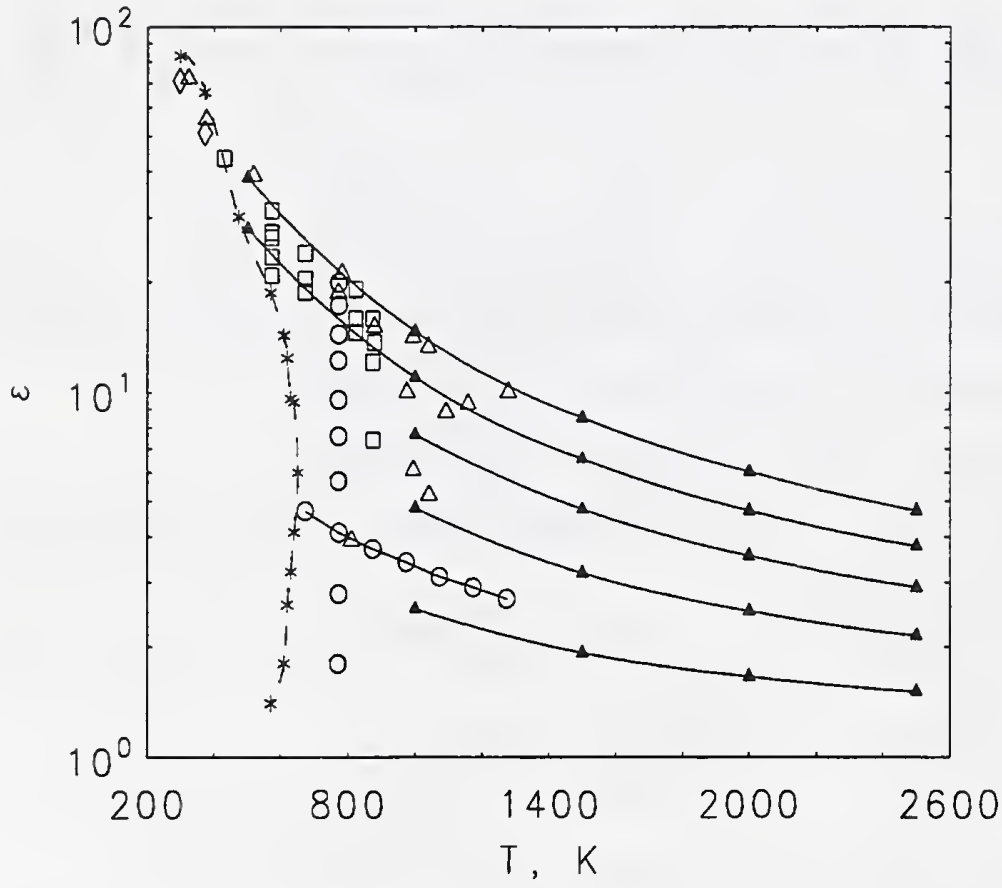

Fig 2. The temperature dependence of the SPC/E dielectric constant is shown. The symbols have the same meaning as in Fig. 1. A dashed line joins the points on the SPC/E coexistence curve. ${ }^{13}$ Solid lines join constant density points from a single source. Starting from the bottom of the plot, the solid lines mark densities of $200,300,400,600,800$, and $1000 \mathrm{~kg} / \mathrm{m}^{3}$. 

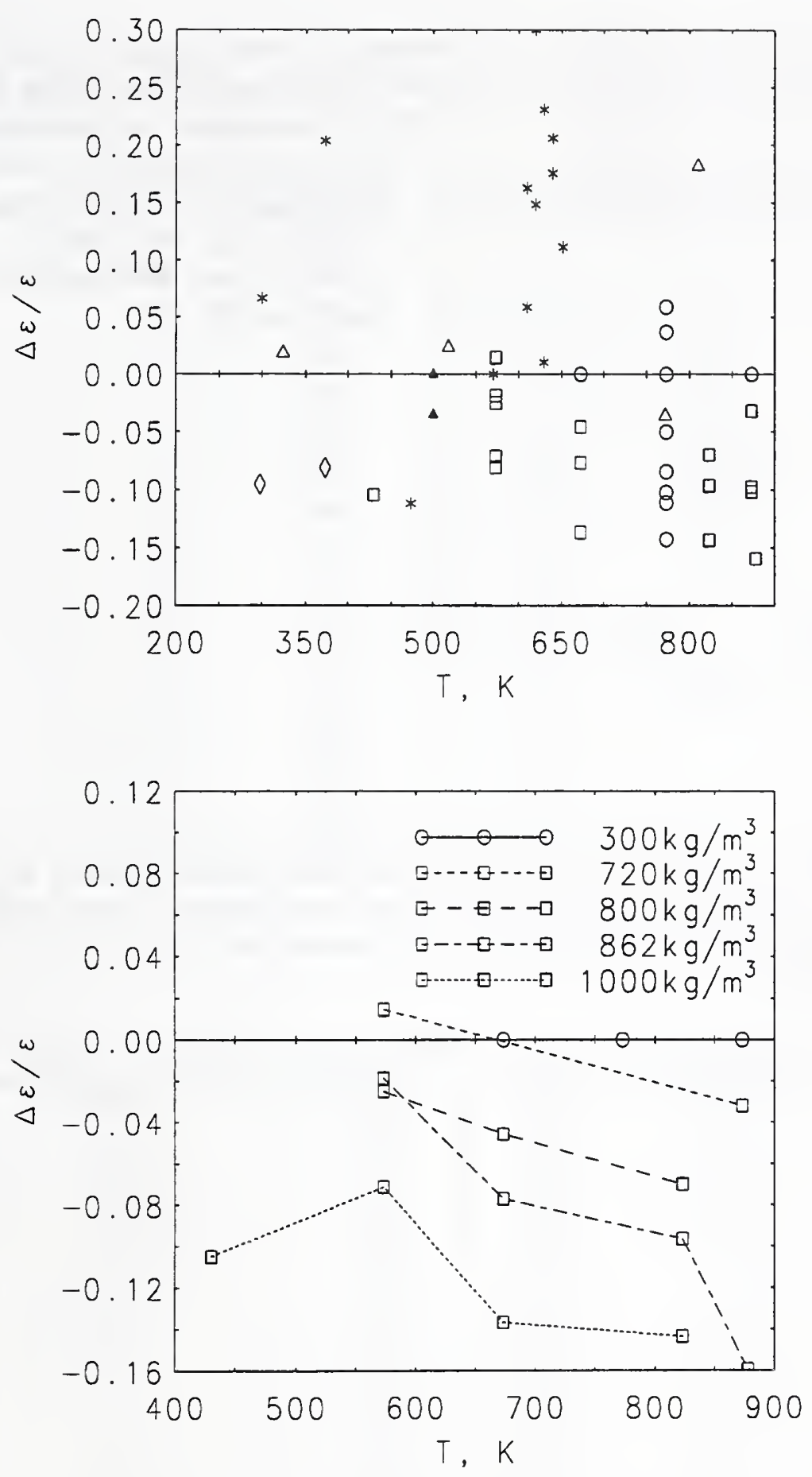

Fig 3. The relative deviations of the simulation values from the correlated experimental values of the dielectric constant are displayed. The symbols have the same meaning as in Fig. 1.
Fig 4. The relative deviations of the simulation values from the correlated experimental values of the dielectric constant are displayed for isochores, as noted in the legend. The symbols have the same meaning as in Fig. 1. 


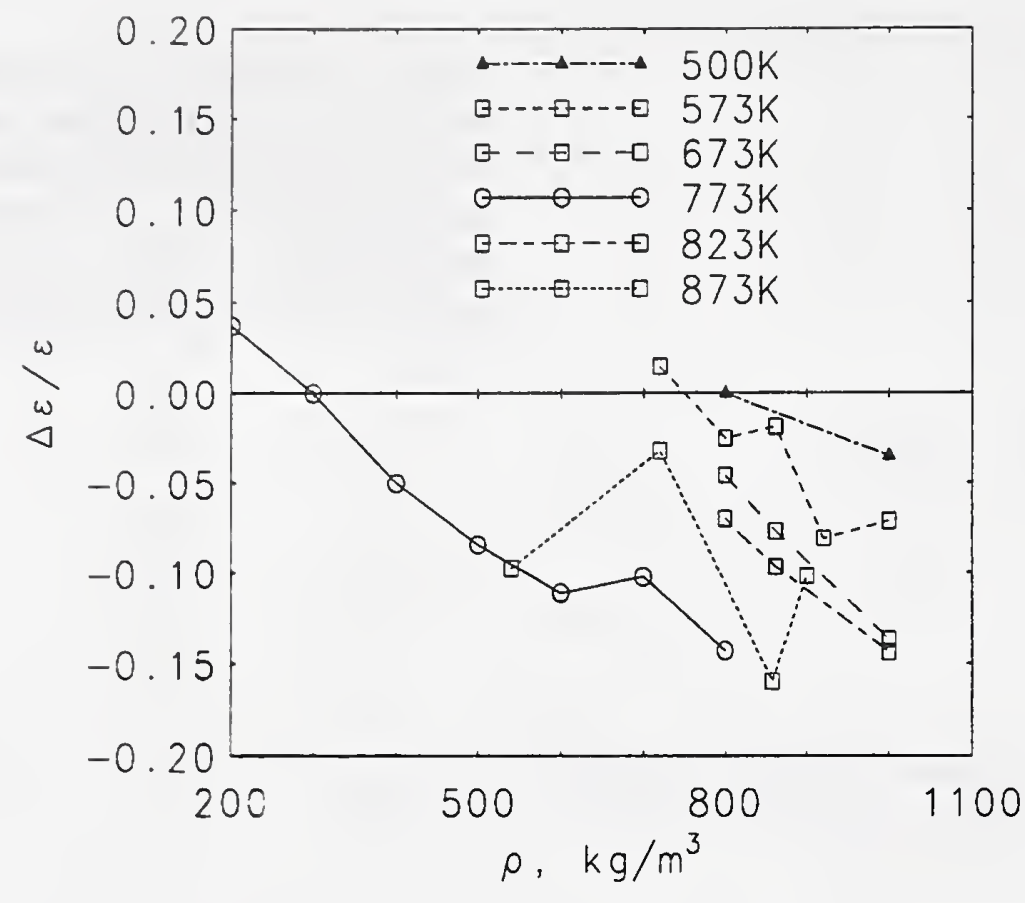

Fig 5. The relative deviations of the simulation values from the correlated experimental values of the dielectric constant are displayed for isotherms, as indicated in the legend. The symbols have the same meaning as in Fig. 1.

Table 1. The dielectric constants, the energy, and the self-diffusion coefficients for SPC/E water produced by RDM are listed for a set of temperatures and densities, along with the values of the dielectric constant of water provided by the correlation. ${ }^{4}$ Values in (...) are extrapolated from the correlation.

\begin{tabular}{|l|c|c|c|c|c|}
\hline $\mathrm{T}, \mathrm{K}$ & $\rho_{m}, \mathrm{~kg} / \mathrm{m}^{3}$ & $\epsilon, \mathrm{SPC} / \mathrm{E}$ & $\epsilon$, corr. & $\mathrm{U}, \mathrm{kJ} / \mathrm{m}$ & $D_{S}, 10^{-9} \mathrm{~m}^{2} / \mathrm{s}$ \\
\hline 430 & 1000. & 43.5 & 48.6 & -34.8 & 10.2 \\
573 & 720. & 20.8 & 20.5 & -25.8 & 31.8 \\
573 & 800. & 23.4 & 24.0 & -27.4 & 27.0 \\
573 & 862. & 26.4 & 26.9 & -28.4 & 23.8 \\
573 & 920. & 27.3 & 29.7 & -29.4 & 21.8 \\
573 & 1000. & 31.3 & $(33.7)$ & -30.4 & 18.1 \\
673 & 800. & 18.8 & 19.7 & -24.6 & 32.7 \\
673 & 862. & 20.4 & 22.1 & -25.8 & 28.8 \\
673 & 1000. & 24.0 & $(27.8)$ & -27.8 & 21.2 \\
823 & 800. & 14.6 & 15.7 & -21.4 & 40.5 \\
823 & 862. & 15.9 & $(17.6)$ & -22.5 & 34.5 \\
823 & 1000. & 19.1 & $(22.3)$ & -24.5 & 26.9 \\
873 & 540. & 7.4 & $(8.2)$ & -15.1 & 65.8 \\
873 & 720. & 12.1 & $(12.5)$ & -19.0 & 49.2 \\
873 & 900. & 15.9 & $(17.7)$ & -22.2 & 34.8 \\
878 & 858. & 13.7 & $(16.3)$ & -21.5 & 38.0 \\
\hline
\end{tabular}


Table 2. The dielectric constants, the energy, and the pressure for SPC/E water produced by AW are listed with the appropriate temperatures and densities. Also listed are the values of the dielectric constant of water provided by the correlation. ${ }^{4}$

\begin{tabular}{|r|c|c|c|c|r|}
\hline $\mathrm{T}, \mathrm{K}$ & $\rho_{m}, \mathrm{~kg} / \mathrm{m}^{3}$ & $\epsilon, \mathrm{SPC} / \mathrm{E}$ & $\epsilon$, corr. & $\mathrm{U}, \mathrm{kJ} / \mathrm{m}$ & $\mathrm{P}, \mathrm{MPa}$ \\
\hline 673 & 300. & 4.7 & 4.7 & -18.4 & 31.5 \\
773 & 100. & 1.8 & 1.7 & -7.6 & 25.0 \\
773 & 200. & 2.8 & 2.7 & -12.4 & 41.5 \\
773 & 300. & 4.1 & 4.1 & -15.8 & 57.0 \\
773 & 400. & 5.7 & 6.0 & -18.6 & 79.0 \\
773 & 500. & 7.6 & 8.3 & -21.1 & 111.5 \\
773 & 600. & 9.6 & 10.8 & -23.4 & 166.0 \\
773 & 700. & 12.3 & 13.7 & -25.4 & 258.0 \\
773 & 800. & 14.4 & 16.8 & -27.5 & 421.0 \\
773 & 900. & 17.3 & & -29.3 & 672.0 \\
773 & 1000. & 19.9 & & -30.7 & 1053.0 \\
873 & 300. & 3.7 & 3.7 & -13.9 & 84.0 \\
973 & 300. & 3.4 & & -12.5 & 109.0 \\
1073 & 300. & 3.1 & & -11.4 & 135.0 \\
1173 & 300. & 2.9 & & -10.5 & 159.0 \\
1273 & 300. & 2.7 & & -9.7 & 184.0 \\
\hline
\end{tabular}


Table 3. The dielectric constants, the energy, and the pressure for SPC/E water determined in Ref. 10 are listed for a set of temperatures and densities. Values of the dielectric constant of water provided by the correlation are limited to $500 \mathrm{~K} .{ }^{4}$

\begin{tabular}{|r|c|c|c|}
\hline $\mathrm{T}, \mathrm{K}$ & $\rho_{m}, \mathrm{~kg} / \mathrm{m}^{3}$ & $\epsilon, \mathrm{SPC} / \mathrm{E}$ & $\epsilon$, corr. \\
\hline 500 & 800. & 28.0 & 28.8 \\
500 & 1000. & 38.6 & 40.0 \\
1000 & 200. & 2.5 & \\
1000 & 400. & 4.8 & \\
1000 & 600. & 7.7 & \\
1000 & 800. & 11.0 & \\
1000 & 1000. & 14.7 & \\
1500 & 200. & 1.9 & \\
1500 & 400. & 3.2 & \\
1500 & 600. & 4.8 & \\
1500 & 800. & 6.6 & \\
1500 & 1000. & 8.5 & \\
2000 & 200. & 1.7 & \\
2000 & 400. & 2.5 & \\
2000 & 600. & 3.5 & \\
2000 & 800. & 4.7 & \\
2000 & 1000. & 6.1 & \\
2500 & 200. & 1.5 & \\
2500 & 400. & 2.1 & \\
2500 & 600. & 2.9 & \\
2500 & 800. & 3.8 & \\
2500 & 1000. & 4.7 & \\
\hline
\end{tabular}


Table 4. The dielectric constants and self-diffusion coefficients quoted in various studies of the SPC/E model. No entry for $D_{S}$ indicates that no value was reported. No entry for $\epsilon$, corr indicates that the state point is outside the range of the correlation. ${ }^{4}$

\begin{tabular}{|c|c|c|c|c|c|}
\hline $\mathrm{T}, \mathrm{K}$ & $\rho_{m}, \mathrm{~kg} / \mathrm{m}^{3}$ & $\epsilon, \mathrm{SPC} / \mathrm{E}$ & $\epsilon$, corr. & $D_{S}, 10^{-9} \mathrm{~m}^{2} / \mathrm{s}$ & Reference \\
\hline 263 & 998. & 112. \pm 16 . & 92.1 & 0.96 & 11 \\
\hline 298 & 998. & 89. \pm 13. & 78.5 & 2.15 & 11 \\
\hline 298 & 998. & $71.0 \pm 6.1$ & 78.5 & & 12 \\
\hline 373 & 958. & 51. \pm 4 & 55.5 & 6.8 & 11 \\
\hline 300 & 998. & 83.1 & 77.9 & 2.6 & 13 \\
\hline 373 & 949. & 66.1 & 54.9 & 7.9 & 13 \\
\hline 473 & 841. & 30.0 & 33.8 & 19.6 & 13 \\
\hline 570 & 36. & 1.4 & & 556.0 & 13 \\
\hline 570 & 674. & 18.7 & 18.7 & 34.7 & 13 \\
\hline 610 & 82. & 1.8 & 1.7 & 238.0 & 13 \\
\hline 610 & 545. & 14.3 & 12.3 & 53.6 & 13 \\
\hline 620 & 112. & 2.6 & 2.0 & 191.0 & 13 \\
\hline 620 & 507. & 12.4 & 10.8 & 54.1 & 13 \\
\hline 630 & 162. & 3.2 & 2.6 & 157.0 & 13 \\
\hline 630 & 470. & 9.6 & 9.5 & 65.6 & 13 \\
\hline 640 & 217. & 4.1 & 3.4 & 129.0 & 13 \\
\hline 640 & 426. & 9.4 & 8.0 & 71.7 & 13 \\
\hline 652 & 326 . & 6.0 & 5.4 & 106.0 & 13 \\
\hline 324 & 1000. & $72.0 \pm 14$ & 70.7 & & 14 \\
\hline 377 & 1111. & $55.7 \pm 4.5$ & & & 14 \\
\hline 518 & 1000. & $39.1 \pm 3.0$ & 38.2 & & 14 \\
\hline 772 & 876. & $18.7 \pm 1.1$ & 19.4 & & 14 \\
\hline 782 & 1000. & $21.1 \pm 1.1$ & & & 14 \\
\hline 810 & 257. & $3.9 \pm 0.2$ & 3.3 & & 14 \\
\hline 878 & 858. & $15.1 \pm 0.9$ & & & 14 \\
\hline 975 & 756. & $10.0 \pm 0.5$ & & & 14 \\
\hline 994 & 500. & $6.1 \pm 0.3$ & & & 14 \\
\hline 994 & 909. & $14.2 \pm 0.9$ & & & 14 \\
\hline 1040 & 1000. & $13.3 \pm 0.8$ & & & 14 \\
\hline 1042 & 429. & $5.2 \pm 0.2$ & & & 14 \\
\hline 1091 & 702. & $8.8 \pm 0.5$ & & & 14 \\
\hline 1158 & 808. & $9.3 \pm 0.3$ & & & 14 \\
\hline 1278 & 1000. & $10.0 \pm 0.6$ & & & 14 \\
\hline
\end{tabular}


Table 5. The potential energy and self-diffusion coefficients quoted in various studies of the SPC/E model. No entry for $U$ or for $D_{S}$ indicates that no value was reported.

\begin{tabular}{|r|c|c|c|c|}
\hline $\mathrm{T}, \mathrm{K}$ & $\rho_{m}, \mathrm{~kg} / \mathrm{m}^{3}$ & $\mathrm{U}, \mathrm{kJ} / \mathrm{mol}$ & $D_{S}, 10^{-9} \mathrm{~m}^{2} / \mathrm{s}$ & Reference \\
\hline 210 & 1024. & -48.01 & 0.05 & 15 \\
220 & 1025. & -46.95 & 0.16 & 15 \\
230 & 1027. & -46.08 & 0.31 & 15 \\
240 & 1026. & -45.08 & 0.50 & 15 \\
250 & 1025. & -44.19 & 0.59 & 15 \\
261 & 1022. & -43.20 & 1.08 & 15 \\
282 & 1016. & -41.28 & 1.51 & 15 \\
307 & 1001. & -39.60 & 2.51 & 15 \\
328 & 987. & -37.26 & 3.57 & 15 \\
263 & 998. & -43.6 & 0.96 & 11 \\
298 & 998. & -41.4 & 2.15 & 11 \\
298 & 998. & -41.5 & & 12 \\
373 & 958. & -37.1 & 6.8 & 11 \\
277 & 990. & & 1.75 & 16 \\
300 & 976. & & 3.18 & 16 \\
306 & 998. & -41.4 & 2.5 & 1 \\
273 & 900. & & 1.36 & 17 \\
273 & 1000. & & 1.46 & 17 \\
300 & 800. & & 2.92 & 17 \\
300 & 900. & & 2.70 & 17 \\
300 & 1000. & & 2.43 & 17 \\
611 & 500. & -20.37 & & 18 \\
996 & 500. & -11.90 & & 18 \\
1979 & 500. & -4.65 & & 18 \\
294 & 1000. & -41.96 & & 18 \\
1038 & 1000. & -21.26 & & 18 \\
1986 & 1000. & -9.86 & & \\
951 & 1500. & -21.68 & & \\
& & & & \\
\hline
\end{tabular}


